\title{
Role of Case Reports in Modern Medical Education
}

\author{
Aftab Merchant and Paul Chastain II* \\ Department of Health Sciences Education, The University of Illinois, College of Medicine at Rockford, USA
}

Clinicians share exciting and novel clinical presentations, procedure modifications, and challenging management issues with their colleagues by reporting them in medical journals as case reports. On many occasions, it includes associations between various conditions and potential differential diagnosis possibilities. For busy clinicians and medical students, case reports serve as a supplementary resource for rapid review of clinical conditions and the variety of different ways they can present.

Journals publish case reports for many reasons including an unusual or novel occurrence of a clinical condition, an unexpected event in the course of treating a patient, an unexpected association between clinical features of diseases, and unique approaches to treat a disease. They act as conduits between peers in the sharing of information and contribute to evidence-based medicine and medical research.

In recent years, journals are publishing case reports in formats that best serve the needs of their subscribers. A case report with a brief clinical presentation and short questions and answers is a more interactive format that keeps the reader engaged. Smith [1] reported a case of conjunctivitis followed by five short questions and answers that highlight key points to consider in history taking, diagnosis, and management of the patient. This approach is in contrast to the traditional case reports that provide a detailed history, physical findings, test results, differential diagnosis, and clinical course and outcome of the case followed by a discussion on the relevant features of the case and how this case differs from those that are reported in the literature. Case reports published by Faruqui [2,3] and Lurie [4] are examples of such formats with the clinical problem-solving approach and relevant commentary. Some journals have opted for a middle course. Anheier and Schultheis [5] recently reported a De Garengeot hernia in a 68-year-old woman. They provided a brief presentation of the case followed by a more detailed discussion.

The role of case reports in medical education goes beyond just suggesting student subscribe to and become abreast of the latest novel findings and reviews. They are excellent teaching and learning tools that can be used wisely in both preclinical and clinical training of the medical students. The clinical findings and the given data from a patient can be used in the classrooms to help students interpret the data to formulate a differential diagnosis and plan management strategies. They can then compare their approach to the patient's condition to the authors and discuss their merits.

Medical schools are now shifting from a traditional disciplinebased approach towards a more integrated, system-based, clinically relevant curriculum. Case-based learning, problem-based-learning, and team-based learning are new active learning formats that rely heavily on the application of foundational concepts towards real-world scenarios. Case reports are an excellent resource for medical educators to bring original clinical cases in their active learning sessions and train students based on their level of experience to interpret data and solve problems. Students can see the degree to which they have learned dovetails with what is needed to address these challenging but rewarding clinical cases. The ability to see gaps and fill in these gaps is critical for them to be able to be lifelong learners and also to tackle and master the independent learning that is expected during med school.

One of the best uses of case reports is in the team-based learning model of instructions. Team-based learning (TBL) prepares students for the class through a readiness assurance process and then facilitates learning through active learning application exercises to solve complex problems. Students are assigned preparation materials to review before the class. In the context of case report based TBLs, this material would be relevant molecular, biochemical, physiological processes that occur in a normal, healthy individual, various exogenous and endogenous triggers that cause the transition from normal to a pathological condition, appropriate diagnostic testing modalities, typical patient presentations, and potential treatment options. The readiness assurance process of team-based learning allows students to determine how well they understood and integrated the preparation material and to clarify any misunderstanding or confusion concerning that material. The application exercises enable teams to learn how to work as a cohesive group and come to a consensus. After the teams have come up with their solutions, they discuss the rationale for their decisions in class discussions facilitated by the subject experts. Case reports due to the nature of their atypical presentations, approaches, and outcomes challenge teams to build upon the pre-class material and expand further to look for diagnosis and treatment options for the reported problems.

Simulation labs are another area of modern medical education where case reports are particularly useful. Simulation labs provide the opportunities for students to learn and improve their skills in clinical data interpretation, examination skills, and technical procedures. Depending on the educational experience and level of training, a case report can be used in its original form or modified for simulation lab experiences. In our simulations, students meet their patient (typically a mannequin that has been programmed to speak and interact with the students) and listen to the patient describe why they are there. The case studies allow us to envision the original dialog between the authors and their patient to allow our mannequin-based simulation to be as authentic as possible. The students go through the simulation to formulate a differential diagnosis, review test results, reach a diagnosis, and determine appropriate treatment options. During the debrief,

${ }^{*}$ Correspondence to: Paul Chastain, Department of Health Sciences Education, The University of Illinois, College of Medicine at Rockford, 1601 Parkview Avenue, Rockford, Il 61107, USA, Tel: 815-395-5622; E-mail: pdchast7@uic.edu

Received: June 20, 2018; Accepted: June 25, 2018; Published: June 30, 2018 
students are given the original case report to compare their problemsolving rationale with those of the individuals working in the field. The case reports provide feedback and a unique opportunity for them to learn and improve.

Case reports not only allows students to review a clinical condition but also provides opportunities to learn writing case presentations and physical findings for documentation purposes. Skills that will go a long way in their careers to clearly and methodically communicate relevant patient information to colleagues and health professionals in other disciplines.

For young researchers, case reports are a great venue to begin research and medical writing. Writing case report allows deep learning of the condition, opportunities to review the work of other researchers, and present their point of view in a meaningful way without the pressures of an extended research protocol.

In summary, case reports are of value in the modern medical education because they can be used throughout the curriculum (e.g., case-based, problem-based, and team-based learning, simulation labs, learning documentation skills, and research). Also, with schools moving towards integrated and applied curriculums, it encourages medical schools to seek additional ways to utilize case studies and reports to meet the needs of a new generation of medical educators training the next generation of doctors.

\section{References}

1. Staunton Smith T (2018) A tricky sticky eye. Aust J Gen Pract 47: 286-287. [Crossref]

2. Farooqui AN, Merchant A, Ahmed L (2000) Klebsiella rhinoscleromatis--an innocent or a deadly organism. J Pak Med Assoc 50: 395-397. [Crossref]

3. Farooqui AN, Merchant A, Nabeel I (2000) Henoch Schonlein Purpura associated with Helicobacter pylori infection and Duodenal Ulcer. J Coll Physicians Surg Pak 10: $80-82$.

4. Lurie JD, Gerber PD, Sox HC (2000) Clinical problem-solving. A pain in the back. $N$ Engl J Med 343: 723-726. [Crossref]

5. Anheier M, Schultheis KH (2018) De Garengeot hernia - Uncommon cause of a right groin pain. Clin Case Studie Rep 1: 1-3.

Copyright: $\mathbb{C} 2018$ Merchant A. This is an open-access article distributed under the terms of the Creative Commons Attribution License, which permits unrestricted use, distribution, and reproduction in any medium, provided the original author and source are credited. 